\title{
Extended Object and Group Tracking with Elliptic Random Hypersurface Models
}

\author{
Marcus Baum, Benjamin Noack, and Uwe D. Hanebeck \\ Intelligent Sensor-Actuator-Systems Laboratory (ISAS), \\ Institute for Anthropomatics, \\ Karlsruhe Institute of Technology (KIT), Germany. \\ \{marcus.baum, benjamin.noack\}@kit.edu, uwe.hanebeck@ieee.org
}

\begin{abstract}
This paper provides new results and insights for tracking an extended target object modeled with an Elliptic Random Hypersurface Model (RHM). An Elliptic RHM specifies the relative squared Mahalanobis distance of a measurement source to the center of the target object by means of a one-dimensional random scaling factor. It is shown that uniformly distributed measurement sources on an ellipse lead to a uniformly distributed squared scaling factor. Furthermore, a Bayesian inference mechanisms tailored to elliptic shapes is introduced, which is also suitable for scenarios with high measurement noise. Closed-form expressions for the measurement update in case of Gaussian and uniformly distributed squared scaling factors are derived.
\end{abstract}

Keywords: Tracking, extended objects, random hypersurface models.

\section{Introduction}

In tracking applications, a target object is considered as extended if the received measurements may stem from different locations, named measurement sources, on the target object. This paper is concerned with tracking a single extended target object. We assume that the measurement sources are unknown and no association of measurements to measurement sources is available. Such tracking problems arise for instance in air surveillance scenarios [1], where aircraft shall be tracked by means of high-resolution radar devices (see Fig. 1). Due to the resolution capability of the radar device, several measurements may be received from different scattering centers on the aircraft. In order to improve the robustness and precision of the estimation results, it is desired to estimate the target extent in addition the target position.

A second important application (see Fig. 1) is tracking a collectively moving group of point targets [1]. If the point targets are closely spaced compared to the sensor noise, it becomes hard to perform data association. In this case, it is suitable to consider this

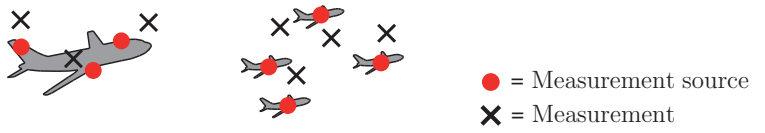

Figure 1: Extended object and group of targets.

group of point targets as a single extended object (see Fig. 1). Note that tracking multiple extended objects is not treated in this paper.

In this paper, the true shape of the target object is approximated with an ellipse. Elliptic shapes are highly relevant for real world applications, as ellipse provides useful information about the target orientation and extent. The parameters of the ellipse describing the target are unknown and must be estimated based on the received measurements. For this purpose, it is necessary to model the elliptic target extent. We employ a socalled Random Hypersurface Model (RHM), which was already introduced in [2] for arbitrary shapes. An RHM for elliptic shapes specifies the relative squared Mahalanobis distance of a measurement source to the center of the target object by means of a one-dimensional random scaling factor.

This paper provides new results and insights on $E l$ liptic RHMs. When modeling an extended target with an Elliptic RHM, one open degree of freedom is to select a proper probability distribution for the random scaling factor. This paper gives a rigorous answer to this question. If no information about the extended target object is available, a uniformly distributed squared scaling factor is suitable. The second major contribution of this paper is the derivation of closed-form expressions for the measurement update for both Gaussian and uniformly distributed squared scaling factors. These results render Elliptic RHMs a promising and practicable approach for tracking extended targets. 


\section{Problem Formulation}

We treat the problem of tracking the position and shape of an unknown extended object in a plane ${ }^{1}$ based on position measurements corrupted by additive stochastic noise. A generic forward model of the generation process of a single measurement is illustrated in Fig. 2. It models how a measurement is generated if the true shape is given. The task of a state estimator is to perform backward inference, i.e., given the measurement it aims at inferring the true shape (the hidden state). Note that the generative forward model is only a model of the reality, it is never actually computed. It only builds the theoretical basis for constructing a state estimator.

In general, the forward model for extended objects consists of two steps: For a given ellipse, first a measurement source is generated (see the first component in Fig. 2). Second, the measurement is generated based on the measurement source according to the measurement model (see Fig. 2). The measurement model usually results from the particular sensor and it is corrupted with stochastic noise.

We assume that the generation of each single measurement is independent. Hence, w.l.o.g. we can assume that at each time step $k$, a single two-dimensional position measurement $\hat{z}_{k}$ is available. The case of several measurements per time step can be treated as a special case. The unknown measurement source at time step $k$ is denoted with $\underline{\tilde{z}}_{k}$. In this paper, the measurement $\underline{\hat{z}}_{k}$ is the observation of the random variable $\underline{\boldsymbol{z}}_{k}$ according to a specific measurement model (see Fig. 2) given by

$$
\underline{\boldsymbol{z}}_{k}=\underline{\tilde{z}}_{k}+\underline{\boldsymbol{v}}_{k}
$$

where $\underline{\boldsymbol{v}}_{k}$ denotes additive white Gaussian observation noise. ${ }^{2}$ The statistics of $\underline{\boldsymbol{v}}_{k}$ are assumed to be known as they result from the particular sensor like a radar device. The location of the measurement source $\underline{\tilde{z}}_{k}$ is unknown. It is modeled with the measurement source model (see Fig. 2).

We aim at developing a Bayesian state estimator for the five-dimensional state vector $\underline{p}_{k-1}$ of the ellipse, which consists of the center and shape. ${ }^{3}$ In the following, we denote the probability density for the parameter vector $\underline{p}_{k-1}$ (the unknown state) after the incorporation of the measurements $\underline{\hat{z}}_{1}, \ldots, \underline{\hat{z}}_{k-1}$ with $f^{e}\left(\underline{p}_{k-1}\right)$.

The state vector is assumed to evolve according to a known Markov model characterized by the conditional density function $f\left(\underline{p}_{k} \mid \underline{p}_{k-1}\right)$. The predicted probability density at time step $k$ thus results from the Chapman-

\footnotetext{
${ }^{1} \mathrm{An}$ extension of the presented results to higher dimensions is straightforward.

${ }^{2}$ All random variables are printed bold face in this paper.

${ }^{3}$ In real world applications, the state vector would also include information about the current velocity and acceleration, for instance. The state vector can easily be extended with further state variables.
}

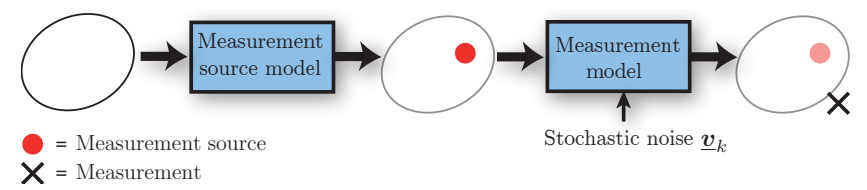

Figure 2: Model of the generation process for one measurement.

Kolomogorov equation

$$
f^{p}\left(\underline{p}_{k}\right)=\int f\left(\underline{p}_{k} \mid \underline{p}_{k-1}\right) \cdot f^{e}\left(\underline{p}_{k-1}\right) d \underline{p}_{k-1} .
$$

The predicted probability density $f^{p}\left(\underline{p}_{k}\right)$ is updated with the next measurement $\underline{\hat{z}}_{k}$ according to Bayes' rule

$$
f^{e}\left(\underline{p}_{k}\right):=c_{k} \cdot f^{L}\left(\underline{\hat{z}}_{k} \mid \underline{p}_{k}\right) \cdot f^{p}\left(\underline{p}_{k}\right),
$$

where $f^{L}\left(\underline{\hat{z}}_{k} \mid \underline{p}{ }_{k}\right)$ is a likelihood function and $c_{k}$ is a normalization factor. Note that in this paper we assume all probability densities to be Gaussian, i.e., $f^{e}\left(\underline{p}_{k}\right) \approx$ $\mathcal{N}\left(\underline{p}_{k}-\underline{\mu}_{k}^{e}, \mathbf{C}_{k}^{e}\right)$ and $f^{p}\left(\underline{p}_{k}\right) \approx \mathcal{N}\left(\underline{p}_{k}-\underline{\mu}_{k}^{p}, \mathbf{C}_{k}^{p}\right)$.

\section{Related Work}

In this section, we give a brief overview of related methods for extended object tracking.

A common approach for modeling extended targets are so-called spatial distribution models $[3,4]$, where each measurement source is assumed to be an independent random draw from a probability distribution. In real world applications, it is difficult to determine a reasonable spatial distribution, since the spatial distribution highly depends on the (unknown) properties of the extended object. Furthermore, a spatial distribution in general depends on the parameter vector of the target shape. Hence, a spatial distribution model can be seen as a hierarchical probability model for which often no closed-form solutions exist. In [1], an elliptic object extension is modeled with a random matrix that is treated as an additional state variable. In this case, it is possible to derive closed-form expressions $[1,5,6]$.

A recent approach $[7,8]$ is based on combined settheoretic and stochastic fusion. There, it is only assumed that the measurement sources lie on the target surface and no (statistical) assumptions are made. Then, it is necessary to assume that the number of measurements, which are received at a particular time step depends on the size of the extended object in order to estimate the size of the object.

Apart from the above approaches, which implicitly model measurement sources, there exist also approaches that explicitly model measurement sources on the target surface [9]. These approaches require data association and are not treated in this paper. 


\section{Elliptic RHMs}

An Elliptic RHM [2] is a specific measurement source model (see Fig. 2) for extended targets. It specifies the generation of a single measurement source in two steps. For the given true ellipse, first a scaled version of the ellipse is generated, while leaving the center unchanged. The scaling factor is specified by an independent random draw from a one-dimensional probability density function (see the red-colored function in Fig. 3). Second, the measurement source is selected from the scaled ellipse according to an arbitrary, unknown rule. Note that the random scaling factor can be interpreted as the (relative) distance of the measurement source from the target center with respect to the squared Mahalanobis distance induced by the true ellipse.

The probability density of the scaling factor has to be specified in advance. Together with the elliptic shape function, it forms the target model. The scaling factor can be assumed to be independent of the target shape. It is important to note that all five ellipse parameters can be estimated based on measurements generated from an Elliptic RHM.

In the following, several properties and advantages of Elliptic RHMs are summarized:

- $R H M s$ do not require a hierarchical probability model. Backward inference with RHMs is similar to shape fitting. In this paper, we even show that for Gaussian and uniform distributed squared scaling factors, closed-form expressions can be derived (see Section 4.3).

- Since the parameters of the ellipse are modeled with a multivariate Gaussian distribution, Elliptic $R H M s$ can easily be embedded into other Bayesian inference algorithms, e.g., data association, retrodiction, and interacting multiple models (IMMs).

- An RHM does not impose strong restrictions on the measurement sources on the target object. Therefore, this approach is robust to systematic errors in the target model, i.e., when the true shape and distribution of the measurement sources do not coincide with the modeled shape (see Section 4.2).

- If the measurement sources are uniformly distributed on the ellipse, an Elliptic RHM with a uniformly distributed squared scaling factor is able to estimate the correct ellipse. Note that there are no closed-form expressions for a uniform spatial distribution $[3,4]$ on an ellipse available. However, a uniform distribution on an ellipse can be approximated with a Gaussian distribution [6].

- The shape of a target object can be estimated correctly even if only one measurement per time step is available and the object is static.

- RHMs are modular. The parametric representation of the shape, and the inference mechanism can be changed. RHMs can even be generalized to other shapes.

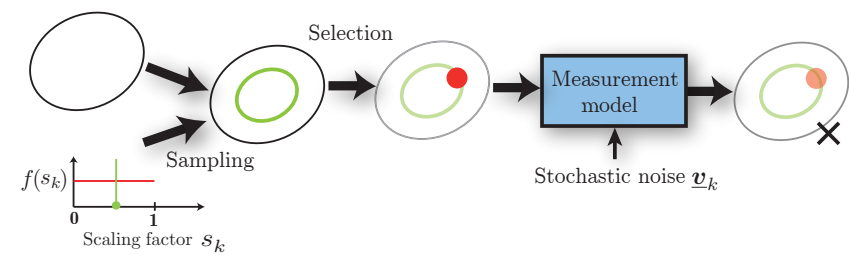

Figure 3: Random Hypersurface Models.

\subsection{Formalization}

In order to give a formal definition of Elliptic RHMs, a suitable parametric representation of an ellipse has to be selected. We will select this representation such that we obtain a polynomial shape function. In this case, the first two moments of the measurement update can be calculated analytically.

Definition 1 (Ellipse). A two-dimensional ellipse with center $\underline{m}_{k}$ and positive semi-definite shape matrix $\mathbf{A}_{k}$ is given by $\left\{\underline{z} \mid \underline{z} \in \mathbb{R}^{2}\right.$ and $\left.\left(\underline{z}-\underline{m}_{k}\right)^{T} \mathbf{A}_{k}^{-1}\left(\underline{z}-\underline{m}_{k}\right) \leq 1\right\}$.

In order to avoid the treatment of positive semidefinite random matrices, we directly employ the Cholesky decomposition $\left(\mathbf{A}_{k}\right)^{-1}=\mathbf{L}_{k} \mathbf{L}_{k}^{T}$, where

$$
\mathbf{L}_{k}:=\left[\begin{array}{cc}
a_{k} & 0 \\
c_{k} & b_{k}
\end{array}\right]
$$

is a lower triangular matrix with positive diagonal entries. The parameter vector of an ellipse is then given by $\underline{p}_{k}=\left[\underline{m}_{k}^{T}, a_{k}, b_{k}, c_{k}\right]^{T}$, which consists of the center and the non-zero entries of the Cholesky decomposition. With the shape function

$$
g\left(\underline{z}, \underline{p}_{k}\right):=\left(\underline{z}-\underline{m}_{k}\right)^{T} \cdot\left(\mathbf{L}_{k} \mathbf{L}_{k}^{T}\right) \cdot\left(\underline{z}-\underline{m}_{k}\right),
$$

the ellipse $\mathcal{E}_{k}$ at time step $k$ can be written as

$$
\mathcal{E}_{k}=\left\{\underline{z} \mid \underline{z} \in \mathbb{R}^{2} \text { and } g\left(\underline{z}, \underline{p}_{k}\right)=1\right\} .
$$

The scaled version of $\mathcal{E}_{k}$ with scaling factor $s$ turns out to be

$$
\mathcal{E}_{k}^{s}=\left\{\underline{z} \mid \underline{z} \in \mathbb{R}^{2} \text { and } g\left(\underline{z}, \underline{p}_{k}\right)=s^{2}\right\} .
$$

Now, we are able to formally define an Elliptic RHM.

Definition 2 (Elliptic RHM). Let $\underline{p}_{k}$ contain the parameters of the true ellipse $\mathcal{E}_{k}$ describing an extended object at time step $k$. Let $s_{k}$ be a one-dimensional random variable that models a random scaling factor. The measurement source $\underline{\tilde{z}}_{k}$ is generated according to the Elliptic RHM with scaling factor $\boldsymbol{s}_{k}$ iff

$$
\underline{\tilde{z}}_{k} \in \mathcal{E}_{k}^{\tilde{s}_{k}}
$$

where $\tilde{s}_{k}$ is a realization of the random variable $\boldsymbol{s}_{k}$ and $\mathcal{E}_{k}^{\tilde{s}_{k}}$ is the scaled version of the ellipse $\mathcal{E}_{k}$. An equivalent condition is

$$
g\left(\underline{\tilde{z}}_{k}, \underline{p}_{k}\right)=\tilde{s}_{k}^{2} .
$$




\subsection{Scaling Factors}

In order to employ an Elliptic RHM for extended object tracking, one has to select a proper probability distribution for the random scaling factor in advance, as it is part of the measurement source model. The random scaling factor can be seen as the analogon to a spatial distribution. However, one can say that a random scaling factor in an RHM encompasses many spatial distributions. In the following, we will give further insights on how to select the statistics of the random scaling factor.

If no information about the extended object is available, a natural approach would be to assume the measurement sources to be uniformly drawn from the ellipse. Hence, an obvious question that arises is: If the measurement sources are drawn from a uniform spatial distribution on the entire ellipse surface, what is the probability distribution of the corresponding scaling factor? Obviously, the scaling factor cannot be uniformly distributed as well, because a uniformly distributed scaling factor prefers measurement sources close to the center of the ellipse. However, the following theorem shows that the squared scaling factor is uniformly distributed on the interval $[0,1]$.

Theorem 1. Let $\mathcal{E}_{k}$ be an ellipse specified by the parameter vector $p_{k}$. If the measurement source $\underline{\boldsymbol{z}}$ is drawn uniformly distributed over the ellipse $\mathcal{E}_{k}$, the squared scaling factor $\boldsymbol{s}^{2}=g\left(\underline{\boldsymbol{z}}, \underline{p}_{k}\right)$ is uniformly distributed on the interval $[0,1]$.

Proof. W.l.o.g. we only consider axis-aligned ellipses whose center lies at the origin, since the orientation and center of the ellipse has no influence on the scaling factor. Hence, we obtain

$$
g\left(\underline{\boldsymbol{z}}, \underline{p_{k}}\right)=\frac{\boldsymbol{z}_{1}^{2}}{a_{k}}+\frac{\boldsymbol{z}_{2}^{2}}{b_{k}},
$$

where $a_{k}$ and $b_{k}$ are the semi axes of the ellipse and $\underline{\boldsymbol{z}}=\left[\boldsymbol{z}_{1}, \boldsymbol{z}_{2}\right]^{T}$ is a random variable, which is uniformly distributed on the ellipse.

We denote the cumulative distribution function of the random variable $s^{2}$ with $F_{s^{2}}(u)$. Then, the following holds for $u \in[0,1]$

$$
\begin{aligned}
F_{s^{2}}(u) & =P\left(\left\{g\left(\underline{\boldsymbol{z}}, \underline{p}_{k}\right) \leq u\right\}\right) \\
& =P\left(\left\{\frac{\boldsymbol{z}_{1}^{2}}{\left(a_{k}\right)^{2}}+\frac{\boldsymbol{z}_{2}^{2}}{\left(b_{k}\right)^{2}} \leq u\right\}\right) \\
& =P\left(\left\{\frac{\boldsymbol{z}_{1}^{2}}{\left(\sqrt{u} \cdot a_{k}\right)^{2}}+\frac{\boldsymbol{z}_{2}^{2}}{\left(\sqrt{u} \cdot b_{k}\right)^{2}} \leq 1\right\}\right) \\
& =\frac{1}{A} \cdot \pi \cdot \sqrt{u} \cdot a_{k} \cdot \sqrt{u} \cdot b_{k} \\
& =u,
\end{aligned}
$$

where the area of the ellipse $\mathcal{E}_{k}$ is denoted with $A=$ $\pi \cdot a_{k} \cdot b_{k}$. For $u<0$ we have

$$
F_{s^{2}}(u)=P\left(\left\{g\left(\underline{\boldsymbol{z}}, \underline{p}_{k}\right) \leq u\right\}\right)=0,
$$

because $g\left(\boldsymbol{z}, \underline{p}_{k}\right)>0$ for all $\boldsymbol{z}$. Finally, for $u>1$ we obtain

$$
F_{s^{2}}(u)=P\left(\left\{g\left(\underline{z}, \underline{p}_{k}\right) \leq u\right\}\right)=1 .
$$

As a consequence, the random variable $s^{2}$ is uniformly distributed on the interval $[0,1]$.

The converse of Theorem 1 is not true. A uniformly distributed squared scaling factor does not necessarily lead to a uniform distribution on the entire ellipse. There are many spatial distributions, which yield a uniformly distributed squared scaling factor! This fact is also discussed in Section 5.1 and illustrated in Fig. 5, where two target shapes that lead to uniform scaling factor are depicted. A consequence of this fact is that an Elliptic RHM is more robust, as it captures a broader class of targets.

It may also be justified to select the random scaling factor to be Gaussian distributed [2]. A Gaussian distribution is for instance useful when measurement sources at the border of the ellipse are more likely than measurement sources in the center of the ellipse.

Note that in general it would be possible to estimate the statistics of the random scaling factor together with the shape parameters of the ellipse. However, this has not been investigated so far and is left out for future work.

\subsection{Bayesian Backward Inference}

Having defined the generic forward model for Elliptic $R H M s$, we are now in the position to develop a Bayesian estimator for the parameters of the ellipse. The task of this state estimator is to perform backward inference, i.e., given the measurement it aims at inferring the true shape parameters (the hidden state).

First, it is interesting to note that for a fixed scaling factor, the problem is equivalent to fitting an ellipse to noisy data points. One possible Bayesian solution to this problem based on the Kalman filter can be found in [10], where the elliptic shape function is linearized around the current estimate and the current measurement in order to render the implicit measurement equation to an explicit equation. However, this approach is not suitable for high measurement noise.

In the following, we construct a Bayesian inference mechanism, which is tailored to elliptic shapes. Furthermore, we derive closed-form expressions for the measurement update with uniformly and Gaussian distributed scaling factors.

If there would be no measurement noise, i.e., $\underline{\tilde{z}}_{k}=$ $\underline{\hat{z}}_{k}$, we could immediately write down the measurement equation

$$
g\left(\underline{\tilde{z}}_{k}, \underline{\boldsymbol{p}}_{k}\right)-\boldsymbol{s}_{k}^{2}=0
$$

which maps the unknown parameters $\underline{\boldsymbol{p}}_{k}$ to the pseudomeasurement 0 with additive noise term $s_{k}^{2}$, which is uniformly distributed on the interval $[0,1]$. 


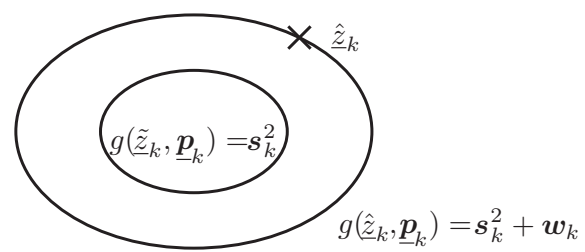

Figure 4: Illustration of the measurement equation.

Unfortunately, the measurement source is not known, only its noisy measurement $\underline{\hat{z}}_{k}=\underline{\tilde{z}}_{k}+\underline{\boldsymbol{v}}_{k}$ is given. If the measurement $\underline{\hat{z}}_{k}$ is inserted in equation (3), a deviation $\boldsymbol{w}_{k}$ on the right hand side may be obtained, i.e., we obtain the measurement equation (see Fig. 4)

$$
g\left(\underline{\hat{z}}_{k}, \underline{\boldsymbol{p}}_{k}\right)-\boldsymbol{s}_{k}^{2}=\boldsymbol{w}_{k}
$$

Actually $\boldsymbol{w}_{k}$ is a random variable, because $\underline{\hat{z}}_{k}$ depends on the measurement noise.

The probability distribution of $\boldsymbol{w}_{k}$ can be approximated with a Gaussian distribution by means of moment matching. For this purpose, the exact first two moments of

$$
\boldsymbol{w}_{k}=g\left(\underline{\tilde{z}}_{k}+\underline{\boldsymbol{v}}_{k}, \underline{p}_{k}\right)-s_{k}^{2}
$$

for given $\underline{p}_{k}, \underline{\tilde{z}}_{k}$, and $s_{k}$ are derived in Theorem 3 in the appendix. The mean of $\boldsymbol{w}_{k}$ is independent of the measurement source $\underline{\tilde{z}}_{k}$ and $s_{k}^{2}$, it only depends on $\underline{p}_{k}$ and the covariance matrix of the noise. We perform the following approximation $\underline{p}_{k} \approx \underline{\mu}_{k}^{p}$, which substitutes the unknown true parameters with its current estimate. The variance of $\boldsymbol{w}_{k}$ depends also on the unknown measurement source $\underline{\tilde{z}}_{k}$ and $s_{k}^{2}$. Hence, we additionally perform the approximations $\underline{\tilde{z}}_{k} \approx \underline{\hat{z}}_{k}$ and $s_{k}^{2} \approx 1$.

Remark 1. Based on the measurement equation (4), we obtain the measurement likelihood

$$
f^{L}\left(\underline{\hat{z}}_{k} \mid \underline{p}_{k}\right):=f_{w_{k}+s_{k}^{2}}\left(g\left(\underline{\hat{z}}_{k}, \underline{p}_{k}\right) \mid \underline{p}_{k}\right),
$$

where $f_{w_{k}+s_{k}^{2}}(\cdot)$ denotes the density of $\boldsymbol{w}_{k}+\boldsymbol{s}_{k}^{2}$. For given parameter $\underline{p}_{k}$ of the ellipse, this conditional density gives the probability for observing a measurement with the deviation $g\left(\underline{\hat{z}}_{k}, \underline{p}_{k}\right)=s_{k}^{2}+w_{k}$. Actually, the likelihood allows for embedding Elliptic RHMs into other probabilistic tracking algorithms, e.g., data association, and interacting multiple models (IMMs). Note that the multi-measurement likelihood is just the product of single measurement likelihoods (5), because we have assumed that the measurements are generated independently.

Based on the measurement equation (4), a measurement update can be performed with a nonlinear state estimator like the Unscented Kalman filter (UKF) [11]. However, we will derive in the following closed-form expressions for the first two moments of the updated estimate.
According to the measurement equation (4), the updated probability density $f^{e}\left(\underline{p}_{k}\right)$ can be computed by considering the random vector

$$
\left[\begin{array}{c}
\underline{\boldsymbol{p}}_{k} \\
g\left(\underline{\hat{z}}_{k}, \underline{\boldsymbol{p}}_{k}\right)-\boldsymbol{w}_{k} \\
\boldsymbol{s}_{k}^{2}
\end{array}\right],
$$

with joint density $f\left(\underline{p}_{k}, h_{k}, s_{k}\right)=f\left(\underline{p}_{k}, h_{k}\right) \cdot f\left(s_{k}\right)$ and $\boldsymbol{h}_{k}:=g\left(\underline{\hat{z}}_{k}, \underline{\boldsymbol{p}}_{k}\right)-\boldsymbol{w}_{k}$. The probability density $f\left(\underline{p}_{k}, h_{k}\right)$ can be approximated with a Gaussian distribution with mean $\left[\left(\underline{\mu}_{k}^{p}\right)^{T}, \mu_{k}^{h}\right]^{T}$ and covariance matrix

$$
\left[\begin{array}{cc}
\mathbf{C}_{k}^{p} & \mathbf{C}_{k}^{p, h} \\
\left(\mathbf{C}_{k}^{p, h}\right)^{T} & \mathbf{C}_{k}^{h}
\end{array}\right]
$$

by means of analytic moment matching. The formulas are given in Theorem 5 in the appendix. The updated estimate can then be written as

$$
f^{e}\left(\underline{p}_{k}\right)=f^{p}\left(\underline{p}_{k} \mid \boldsymbol{h}_{k}=\boldsymbol{s}_{k}^{2}\right)
$$

Now, we have to distinguish whether the squared scaling factor is Gaussian distributed or uniformly distributed:

\section{- Gaussian Distributed Squared Scaling Factor}

In this case the Kalman filter equation leads directly to the updated estimate

$$
\begin{aligned}
\mu_{k}^{e} & =\underline{\mu}_{k}^{p}+\left(\mathbf{C}_{k}^{h}+\mathbf{C}_{k}^{s}\right)^{-1} \cdot \mathbf{C}_{k}^{p} \cdot\left(\mu_{k}^{s}-\mu_{k}^{h}\right) \\
\mathbf{C}_{k}^{e} & =\mathbf{C}_{k}^{p}-\left(\mathbf{C}_{k}^{h}+\mathbf{C}_{k}^{s}\right)^{-1} \cdot\left(\mathbf{C}_{k}^{p}\right)^{2}
\end{aligned}
$$

with $s_{k}^{2} \sim \mathcal{N}\left(\mu_{k}^{s}, \mathbf{C}_{k}^{s}\right)$.

- Uniformly Distributed Squared Scaling Factor If $s_{k}^{2}$ is uniformly distributed on the interval [0,1], we obtain

$$
f^{e}\left(\underline{p}_{k}\right)=d_{k} \int f^{*}\left(\underline{p}_{k}, h_{k}\right) d h_{k},
$$

where $d_{k}$ is a normalization constant and

$$
f^{*}\left(\underline{p}_{k}, h_{k}\right):= \begin{cases}f\left(\underline{p}_{k}, \underline{h}_{k}\right) & \text { if } 0 \leq \underline{h}_{k} \leq 1 \\ 0 & \text { otherwise }\end{cases}
$$

is a truncated Gaussian distribution. The first two moments of $f^{e}\left(\underline{p}_{k}\right)$ can be computed analytically by means of Lemma 2 .

\section{Simulation}

In this section, the practicability of Elliptic RHMs is demonstrated by means of two example scenarios. 


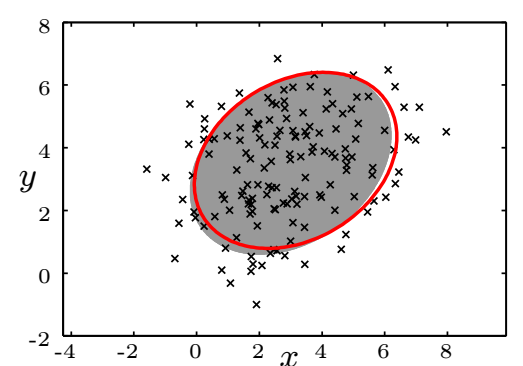

(a) The extended object is in fact an ellipse. The covariance matrix of the measurement noise is $\operatorname{diag}(1,1)$.

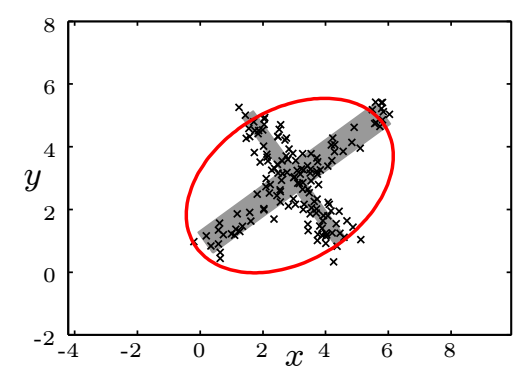

(b) The extended object consists of two rectangles. The covariance matrix of the measurement noise is $\operatorname{diag}(0.1,0.1)$.

Figure 5: Estimating the extent of a static object: The measurement sources are uniformly distributed on the target extent (grey surface). A single measurement (crosses) is received at each time step. The estimated ellipse is plotted in red. The prior Gaussian distribution of the parameters has a mean of $[2,2,0.5,0.5,0]^{T}$ and covariance matrix $\operatorname{diag}\left([5,5,0.02,0.02,0.04]^{T}\right)$.

\subsection{Static Extended Objects}

The first scenario is an extended object that neither moves nor changes its shape and position over time. Furthermore, a single position measurement is received at each time step. In Fig. 5, two extended objects with different shapes are estimated.

The first extended object (Fig. 5a) is in fact an ellipse on which the measurement sources are uniformly distributed. According to Theorem 1, this ellipse can be estimated with a uniformly distributed squared scaling factor. This example demonstrates that an Elliptic RHMs is in general capable of estimating the correct parameters of an ellipse in case of uniformly distributed measurement sources.

The shape of the second extended object (see Fig. 5b) is similar to the shape of an airplane. This shape also leads (approximately) to a uniformly distributed squared scaling factor. Hence, the true parameters of the smallest enclosing ellipse of the target object can also be estimated with an Elliptic RHM. The special thing about this example is that the estimator does not know the true shape, however, an Elliptic RHM is still able to estimate the smallest enclosing ellipse of the target object.

\subsection{Group Tracking}

The second scenario shows that Elliptic RHMs are suitable for tracking a group of closely spaced targets. In this scenario, nine individual group members that are arranged in fixed relative positions perform a $45^{\circ}$ turn. The measurement noise is zero-mean Gaussian with covariance $\operatorname{diag}(0.8,0.8)$. At each time step, a measurement is received from each group member. In this scenario, tracking each single group member would be quite difficult, because of the large number of closely spaced targets. Hence, it is suitable to consider this group as one single extended object.

In order to track the elliptic shape of the group with an Elliptic RHM, we employ a constant velocity model for the group motion. Hence, the state vector to be estimated consists of the five parameters for the ellipse and a two-dimensional velocity vector for the center of the ellipse. The additive system noise of the velocity vector is assumed to be zero-mean with covariance matrix $\operatorname{diag}([0.25,0.25])$. The covariance matrix for the uncertainty of the target shape parameters (2) is increased at each time step with $\operatorname{diag}([0.0004,0.0004,0.0012])$ in order to capture shape changes.

Initially, the target is assumed to be located at the position $[0.7,0.7]^{T}$ with an uncertainty given by the covariance matrix $\operatorname{diag}([0.08,0.08])$. The shape parameters of the ellipse $(2)$ are set to $[0.3,0.3,0]^{T}$ with covariance matrix $\operatorname{diag}([0.008,0.008,0.0016])$. Furthermore, the velocity vector is a priori assumed to be $[8,0]^{T}$ with covariance matrix $\operatorname{diag}([0.1,0.3])$.

It can be seen in Fig. 6 that the shape, i.e., the smallest enclosing ellipse, of the group is tracked quite well. In particular, the $45^{\circ}$ turn is detected and shape changes are tracked correctly.

\section{Conclusions and Outlook}

In this paper, we have presented new results on extended object tracking with Elliptic RHMs. We have shown that an uniform distribution on an ellipse leads to a uniformly distributed squared scaling factor. Furthermore, we have provided closed-form expressions for the measurement update with Elliptic RHMs. Simulations have demonstrated that these results render $\mathrm{El}$ liptic RHMs a practicable method for extended object tracking. Future work concentrates on embedding Elliptic RHMs into other tracking algorithms like data association and IMMs. A further application area of Elliptic RHMs may be computer vision, where similar problems arise when tracking and detecting elliptical shapes in images. 


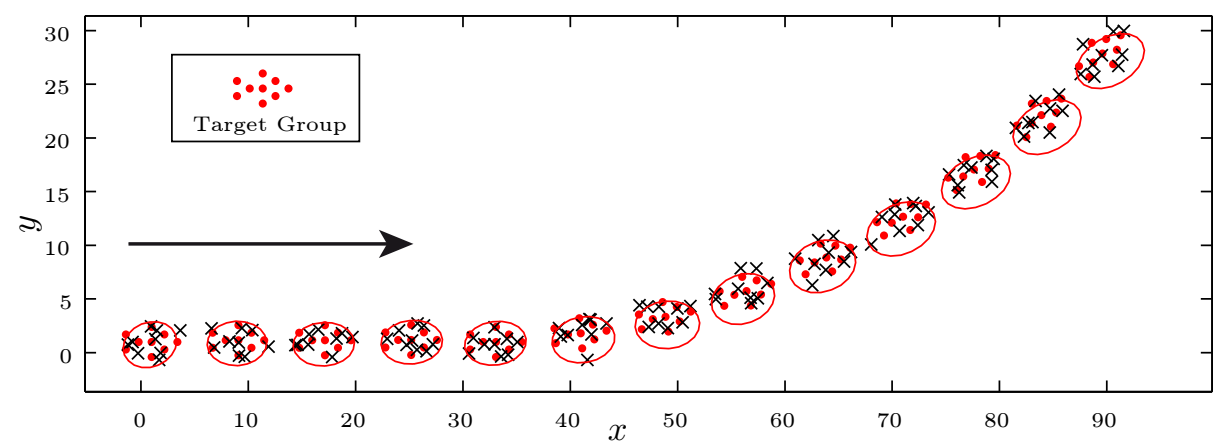

Figure 6: Tracking a group of point targets: Point targets (red dots), measurements (crosses), and estimated ellipse (red) plotted for several time steps.

\section{Appendix}

Theorem 2. For the Gaussian distributed random vector $\left[\boldsymbol{z}_{1}, \ldots, \boldsymbol{z}_{2}\right]^{T} \sim \mathcal{N}(\mu, \Sigma)$ and nonnegative integers $s_{1}$ to $s_{n}$, the following holds [12]:

$$
\begin{aligned}
& \mathrm{E}\left[\prod_{i=1}^{n} \boldsymbol{z}_{i}^{s_{i}}\right]=\sum_{\nu_{1}=0}^{s_{1}} \ldots \sum_{\nu_{n}=0}^{s_{n}} \sum_{r=0}^{[s / 2]}(-1)^{\sum_{i=1}^{n} \nu_{i}} . \\
&\left(\begin{array}{c}
s_{1} \\
\nu_{n}
\end{array}\right) \ldots\left(\begin{array}{c}
s_{n} \\
\nu_{n}
\end{array}\right) \frac{\left(\underline{h}^{T} \Sigma \underline{h}\right)^{r}\left(\underline{h}^{T} \mu\right)^{s-2 r}}{r !(s-2 r) !}
\end{aligned}
$$

where $s=s_{1}+\ldots+s_{n}$ and $\underline{h}=\left[\frac{s_{1}}{2}-\nu_{1}, \ldots, \frac{s_{n}}{2}-\nu_{n}\right]^{T}$.

Theorem 3. W.l.o.g. we assume that the ellipse is centered at the origin with parameter vector $\underline{p}=$ $[0,0, a, b, c]^{T}$. Let $\underline{\boldsymbol{z}}=[\boldsymbol{x}, \boldsymbol{y}]^{T}:=\underline{\tilde{z}}+\underline{\boldsymbol{v}}$ be a Gaussian distributed random variable, where $\underline{\boldsymbol{v}}$ denotes additive white observation noise with covariance matrix $\mathbf{C}^{v}=\left[\begin{array}{cc}c_{x} & c_{x y} \\ c_{x y} & c_{y}\end{array}\right]$. If $\underline{\tilde{z}}$ is known to fulfill the relation $g(\underline{\tilde{z}}, \underline{p})-s^{2}=0$ for a fixed scaling factor $s$, the first two moments of the random variable

$$
g(\underline{z}, \underline{p})-s:=\boldsymbol{w}
$$

are given by

- $\mathrm{E}[\boldsymbol{w}]=\bar{a} c_{x}+2 \bar{c} c_{x y}+\bar{b} c_{y}$

- $\operatorname{Var}[\boldsymbol{w}]=\mathrm{E}\left[\left(g(\underline{\boldsymbol{z}}, \underline{p})-s^{2}\right)^{2}\right]-\mathrm{E}[\boldsymbol{w}]$

$$
\begin{aligned}
= & \mathrm{E}\left[s^{4}-2 \bar{a} s^{2} \boldsymbol{x}^{2}+\bar{a}^{2} \boldsymbol{x}^{4}-4 \bar{c} s^{2} \boldsymbol{x} \boldsymbol{y}+4 \bar{a} \bar{c} \boldsymbol{x}^{3} \boldsymbol{y}-\right. \\
& \left.2 \bar{b} s^{2} \boldsymbol{y}^{2}+\left(2 \bar{a} \bar{b}+4 \bar{c}^{2}\right) \boldsymbol{x}^{2} \boldsymbol{y}^{2}+4 \bar{b} \bar{c} \boldsymbol{x} \boldsymbol{y}^{3}+\bar{b}^{2} \boldsymbol{y}^{4}\right]
\end{aligned}
$$

where $\bar{a}=a^{2}, \bar{c}=a c$ and $\bar{b}=c^{2}+b^{2}$. They can be computed with the help of Theorem 2.
Lemma 1. The following integrals can be computed with the help of basic integral rules:

$$
\begin{aligned}
\phi_{\sigma^{2}}^{0}(b) & :=\int_{-\infty}^{b} \mathcal{N}\left(0, \sigma^{2}\right) \mathrm{d} x=\frac{1}{2}\left(1+\operatorname{erf}\left(\frac{b}{\sqrt{2} \sigma}\right)\right), \\
\phi_{\sigma^{2}}^{1}(b) & :=\int_{-\infty}^{b} x \mathcal{N}\left(0, \sigma^{2}\right) \mathrm{d} x=-\sigma^{2} \mathcal{N}\left(0, \sigma^{2}\right), \\
\phi_{\sigma^{2}}^{2}(b) & :=\int_{-\infty}^{b} x^{2} \mathcal{N}\left(0, \sigma^{2}\right) \mathrm{d} x=b \phi_{\sigma^{2}}^{1}(b)+\sigma^{2} \phi_{\sigma^{2}}^{0}(b) .
\end{aligned}
$$

Theorem 4. Let $\underline{\boldsymbol{x}} \sim \mathcal{N}(\underline{0}, \mathbf{C})$ be a Gaussian distributed $n$-dimensional random vector with mean $\underline{0}$ and covariance matrix $\mathbf{C}=\left(c_{i, j}\right)_{i, j=1, \ldots, n}$. Furthermore, let $f\left(\underline{x} \mid\left\{a \leq \boldsymbol{x}_{k} \leq b\right\}\right)$ be the probability density of $\underline{\boldsymbol{x}}$ given that the $k$-th component of $\boldsymbol{x}$ is less than $b$ and greater than a (with $1 \leq k \leq n$ ). Then, the mean $\underline{m}^{z}$ of $f\left(\underline{x} \mid\left\{a \leq \boldsymbol{x}_{k} \leq b\right\}\right)$ is given by

$$
m_{i}^{z}=\frac{1}{\alpha} c_{i, k} c_{k, k}^{-1}\left(\phi_{c_{k, k}}^{1}(b)-\phi_{c_{k, k}}^{1}(a)\right),
$$

where $\alpha:=\phi_{c_{k, k}}^{0}(b)-\phi_{c_{k, k}}^{0}(a)$ is a normalization constant. The covariance matrix $\mathbf{C}^{z}=\left(c_{i, j}^{z}\right)_{i, j=1, \ldots, n}$ of $f\left(\underline{x} \mid\left\{a \leq \boldsymbol{x}_{k} \leq b\right\}\right)$ is determined by

$$
\begin{aligned}
& c_{i, j}^{z}=\frac{1}{\alpha}\left(c_{i, k} c_{j, k} c_{k}^{-2}\left(\phi_{c_{k, k}}^{2}(b)-\phi_{c_{k, k}}^{2}(a)\right)+\right. \\
& \left.\quad\left(c_{i, j}-c_{i, k} c_{j, k} c_{k, k}^{-1}\right)\left(\phi_{c_{k, k}}^{0}(b)-\phi_{c_{k, k}}^{0}(a)\right)\right)-m_{j}^{z} m_{i}^{z} .
\end{aligned}
$$

Proof. Follows from Lemma 1 and the definition of the mean and covariance matrix of a random vector.

Lemma 2. Let $\underline{\boldsymbol{x}} \sim \mathcal{N}(\underline{m}, \mathbf{C})$ be a Gaussian distributed random vector. The first two moments of $f(x \mid\{0 \leq$ $\left.\left.\boldsymbol{x}_{k} \leq 1\right\}\right)$ are given by $\underline{m}^{y}=\underline{m}^{z}+\underline{m}$ and $\mathbf{C}^{y}=\mathbf{C}^{z}$, where the mean $\underline{m}^{z}$ and covariance matrix $\mathbf{C}^{z}$ of $f(x-$ $\left.\underline{m} \mid\left\{-\underline{m}_{k} \leq \boldsymbol{x}_{k} \leq 1-\underline{m}_{k}\right\}\right)$ can be computed with the help of Theorem 4 .

Theorem 5. Given is the following random vector

$$
\left[\begin{array}{c}
\underline{\boldsymbol{p}}_{k} \\
g\left(\underline{\hat{z}}_{k}, \underline{\boldsymbol{p}}_{k}\right)-\boldsymbol{w}_{k}
\end{array}\right]=\left[\boldsymbol{m}_{k}, \boldsymbol{n}_{k}, \boldsymbol{a}_{k}, \boldsymbol{b}_{k}, \boldsymbol{c}_{k}, \boldsymbol{h}_{k}\right]^{T},
$$


with $\boldsymbol{h}_{k}=\boldsymbol{a}_{k}^{2} \overline{\boldsymbol{m}}_{k}^{2}+2 \boldsymbol{a}_{k} \boldsymbol{c}_{k} \overline{\boldsymbol{m}}_{k} \overline{\boldsymbol{n}}_{k}+\left(\boldsymbol{a}_{k}^{2}+\boldsymbol{c}_{k}^{2}\right) \overline{\boldsymbol{n}}_{k}^{2}-\boldsymbol{w}_{k}$, $\overline{\boldsymbol{m}}_{k}=\boldsymbol{m}_{k}-\hat{x}_{k}$, and $\overline{\boldsymbol{n}}_{k}=\boldsymbol{n}_{k}-\hat{y}_{k}$. The mean of this random vector is

$\left[\begin{array}{c}\mu_{k}^{p} \\ \mu_{k}^{h}\end{array}\right]=\left[\mathrm{E}\left[\boldsymbol{a}_{k}^{2} \overline{\boldsymbol{m}}_{k}^{2}+2 \boldsymbol{a}_{k} \boldsymbol{c}_{k} \overline{\boldsymbol{m}}_{k}{\overline{\mu_{k}}}_{k}^{p}+\left(\boldsymbol{b}_{k}^{2}+\boldsymbol{c}_{k}^{2}\right) \overline{\boldsymbol{n}}_{k}^{2}-\boldsymbol{w}_{k}\right]\right]$,

whose last component can be calculated with the help of Theorem 2. The covariance matrix of $\left[\underline{\boldsymbol{p}}_{k}, \boldsymbol{h}_{k}\right]^{T}$ is composed as follows

$$
\left[\begin{array}{cc}
\mathbf{C}_{k}^{p} & \mathbf{C}_{k}^{p, h} \\
\left(\mathbf{C}_{k}^{p, h}\right)^{T} & \mathbf{C}_{k}^{h}
\end{array}\right], \text { with } \mathbf{C}_{k}^{p, h}=\left[\begin{array}{c}
\mathrm{E}\left[\boldsymbol{m}_{k} \boldsymbol{h}_{k}\right]-\mu_{k}^{m} \mu_{k}^{h} \\
\mathrm{E}\left[\boldsymbol{n}_{k} \boldsymbol{h}_{k}\right]-\mu_{k}^{n} \mu_{k}^{h} \\
\mathrm{E}\left[\boldsymbol{a}_{k} \boldsymbol{h}_{k}\right]-\mu_{k}^{a} \mu_{k}^{h} \\
\mathrm{E}\left[\boldsymbol{b}_{k} \boldsymbol{h}_{k}\right]-\mu_{k}^{b} \mu_{k}^{h} \\
\mathrm{E}\left[\boldsymbol{c}_{k} \boldsymbol{h}_{k}\right]-\mu_{k}^{c} \mu_{k}^{h}
\end{array}\right]
$$

and $\mathbf{C}_{k}^{h}=\mathrm{E}\left[\left(\boldsymbol{h}_{k}\right)^{2}\right]-\left(\mu_{k}^{h}\right)^{2}$. The matrices $\mathbf{C}_{k}^{h}$ and $\mathbf{C}_{k}^{p, h}$ can be computed with the help of the following formulas

$$
\begin{aligned}
& \mathrm{E}\left[\boldsymbol{m}_{k} \boldsymbol{h}_{k}\right]= \\
& \mathrm{E}\left[\boldsymbol{a}_{k}^{2} \overline{\boldsymbol{m}}_{k}^{3}+2 \boldsymbol{a}_{k} \boldsymbol{c}_{k} \overline{\boldsymbol{m}}_{k}^{2} \overline{\boldsymbol{n}}_{k}+\boldsymbol{b}_{k}^{2} \overline{\boldsymbol{m}}_{k} \overline{\boldsymbol{n}}_{k}^{2}+\boldsymbol{c}_{k}^{2} \overline{\boldsymbol{m}}_{k} \overline{\boldsymbol{n}}_{k}^{2}-\overline{\boldsymbol{m}}_{k} \boldsymbol{w}_{k}-\right. \\
& \left.\boldsymbol{a}_{k}^{2} \overline{\boldsymbol{m}}_{k}^{2} \hat{x}_{k}-2 \boldsymbol{a}_{k} \boldsymbol{c}_{k} \overline{\boldsymbol{m}}_{k} \overline{\boldsymbol{n}}_{k} \hat{x}_{k}-\boldsymbol{b}_{k}^{2} \overline{\boldsymbol{n}}_{k}^{2} \hat{x}_{k}-\boldsymbol{c}_{k}^{2} \overline{\boldsymbol{n}}_{k}^{2} \hat{x}_{k}+\boldsymbol{w}_{k} \hat{x}_{k}\right] \\
& \mathrm{E}\left[\boldsymbol{n}_{k} \boldsymbol{h}_{k}\right]= \\
& \mathrm{E}\left[\boldsymbol{a}_{k}^{2} \overline{\boldsymbol{m}}_{k}^{2} \overline{\boldsymbol{n}}_{k}+2 \boldsymbol{a}_{k} \boldsymbol{c}_{k} \overline{\boldsymbol{m}}_{k} \overline{\boldsymbol{n}}_{k}^{2}+\boldsymbol{b}_{k}^{2} \overline{\boldsymbol{n}}_{k}^{3}+\boldsymbol{c}_{k}^{2} \overline{\boldsymbol{n}}_{k}^{3}-\overline{\boldsymbol{n}}_{k} \boldsymbol{w}_{k}-\right. \\
& \left.\boldsymbol{a}_{k}^{2} \overline{\boldsymbol{m}}_{k}^{2} \hat{y}_{k}-2 \boldsymbol{a}_{k} \boldsymbol{c}_{k} \overline{\boldsymbol{m}}_{k} \overline{\boldsymbol{n}}_{k} \hat{y}_{k}-\boldsymbol{b}_{k}^{2} \overline{\boldsymbol{n}}_{k}^{2} \hat{y}_{k}-\boldsymbol{c}_{k}^{2} \overline{\boldsymbol{n}}_{k}^{2} \hat{y}_{k}+\boldsymbol{w}_{k} \hat{y}_{k}\right] \\
& \mathrm{E}\left[\boldsymbol{a}_{k} \boldsymbol{h}_{k}\right]= \\
& \mathrm{E}\left[\boldsymbol{a}_{k}^{3} \overline{\boldsymbol{m}}_{k}^{2}+2 \boldsymbol{a}_{k}^{2} \boldsymbol{c}_{k} \overline{\boldsymbol{m}}_{k} \overline{\boldsymbol{n}}_{k}+\boldsymbol{a}_{k}\left(\boldsymbol{b}_{k}^{2}+\boldsymbol{c}_{k}^{2}\right) \overline{\boldsymbol{n}}_{k}^{2}-\boldsymbol{a}_{k} \boldsymbol{w}_{k}\right] \\
& \mathrm{E}\left[\boldsymbol{b}_{k} \boldsymbol{h}_{k}\right]= \\
& \mathrm{E}\left[\boldsymbol{a}_{k}^{2} \boldsymbol{b}_{k} \overline{\boldsymbol{m}}_{k}^{2}+2 \boldsymbol{b}_{k} \boldsymbol{a}_{k} \boldsymbol{c}_{k} \overline{\boldsymbol{m}}_{k} \overline{\boldsymbol{n}}_{k}+\boldsymbol{b}_{k}\left(\boldsymbol{b}_{k}^{2}+\boldsymbol{c}_{k}^{2}\right) \overline{\boldsymbol{n}}_{k}^{2}-\boldsymbol{b}_{k} \boldsymbol{w}_{k}\right] \\
& \mathrm{E}\left[\boldsymbol{c}_{k} \boldsymbol{h}_{k}\right]= \\
& \mathrm{E}\left[\boldsymbol{a}_{k}^{2} \boldsymbol{c}_{k} \overline{\boldsymbol{m}}_{k}^{2}+2 \boldsymbol{a}_{k} \boldsymbol{c}_{k}^{2} \overline{\boldsymbol{m}}_{k} \overline{\boldsymbol{n}}_{k}+\boldsymbol{c}_{k}\left(\boldsymbol{b}_{k}^{2}+\boldsymbol{c}_{k}^{2}\right) \overline{\boldsymbol{n}}_{k}^{2}-\boldsymbol{c}_{k} \boldsymbol{w}_{k}\right] \\
& \text { and } \mathrm{E}\left[\left(\boldsymbol{h}_{k}\right)^{2}\right]= \\
& \quad \mathrm{E}\left[\boldsymbol{a}_{k}^{4} \overline{\boldsymbol{m}}_{k}^{4}+4 \boldsymbol{a}_{k}^{3} \boldsymbol{c}_{k} \overline{\boldsymbol{m}}_{k}^{3} \overline{\boldsymbol{n}}_{k}+2 \boldsymbol{a}_{k}^{2} \boldsymbol{b}_{k}^{2} \overline{\boldsymbol{m}}_{k}^{2} \overline{\boldsymbol{n}}_{k}^{2}+6 \boldsymbol{a}_{k}^{2} \boldsymbol{c}_{k}^{2} \overline{\boldsymbol{m}}_{k}^{2} \overline{\boldsymbol{n}}_{k}^{2}+\right. \\
& \quad 4 \boldsymbol{a}_{k} \boldsymbol{b}_{k}^{2} \boldsymbol{c}_{k} \overline{\boldsymbol{m}}_{k} \overline{\boldsymbol{n}}_{k}^{3}+4 \boldsymbol{a}_{k} \boldsymbol{c}_{k}^{3} \overline{\boldsymbol{m}}_{k} \overline{\boldsymbol{n}}_{k}^{3}+\boldsymbol{b}_{k}^{4} \overline{\boldsymbol{n}}_{k}^{4}+2 \boldsymbol{b}_{k}^{2} \boldsymbol{c}_{k}^{2} \overline{\boldsymbol{n}}_{k}^{4}+2 \\
& \boldsymbol{c}_{k}^{4} \overline{\boldsymbol{n}}_{k}^{4}-2 \boldsymbol{a}_{k}^{2} \overline{\boldsymbol{m}}_{k}^{2} \boldsymbol{w}_{k}-4 \boldsymbol{a}_{k} \boldsymbol{c}_{k} \overline{\boldsymbol{m}}_{k} \overline{\boldsymbol{n}}_{k} \overline{\boldsymbol{w}}_{k}^{2}-
\end{aligned}
$$

which can be computed with the help of Theorem 2.

\section{References}

[1] J. W. Koch, "Bayesian Approach to Extended Object and Cluster Tracking using Random Matrices," IEEE Transactions on Aerospace and Electronic Systems, vol. 44, no. 3, pp. 1042-1059, July 2008.

[2] M. Baum and U. D. Hanebeck, "Random Hypersurface Models for Extended Object Tracking," in
Proceedings of the 9th IEEE International Symposium on Signal Processing and Information Technology (ISSPIT 2009), Ajman, United Arab Emirates, Dec. 2009.

[3] K. Gilholm and D. Salmond, "Spatial Distribution Model for Tracking Extended Objects," IEE Proceedings - Radar, Sonar and Navigation, vol. 152, no. 5, pp. 364-371, October 2005.

[4] K. Gilholm, S. Godsill, S. Maskell, and D. Salmond, "Poisson Models for Extended Target and Group Tracking," in SPIE: Signal and Data Processing of Small Targets, 2005.

[5] M. Feldmann and D. Franken, "Tracking of Extended Objects and Group Targets using Random Matrices - A New Approach," Proceedings of the 11th International Conference on Information Fusion (Fusion 2008), pp. 1-8, July 2008.

[6] - "Advances on Tracking of Extended Objects and Group Targets using Random Matrices," in Proceedings of the 12th International Conference on Information Fusion (Fusion 2009), Seattle, Washington, July 2009.

[7] M. Baum and U. D. Hanebeck, "Extended Object Tracking based on Combined Set-Theoretic and Stochastic Fusion," in Proceedings of the 12th International Conference on Information Fusion (Fusion 2009), Seattle, Washington, July 2009.

[8] — , "Tracking an Extended Object Modeled as an Axis-Aligned Rectangle," in 4th German Workshop on Sensor Data Fusion: Trends, Solutions, Applications (SDF 2009), Lübeck, Germany, October 2009.

[9] J. Vermaak, N. Ikoma, and S. Godsill, "Sequential Monte Carlo Framework for Extended Object Tracking," IEE Proceedings - Radar, Sonar and Navigation, vol. 152 , no. 5 , pp. $353-363$, October 2005.

[10] Z. Zhang, "Parameter Estimation Techniques: A Tutorial with Application to Conic Fitting," Image and Vision Computing, vol. 15, no. 1, pp. $59-76$, 1997.

[11] S. J. Julier and J. K. Uhlmann, "Unscented Filtering and Nonlinear Estimation," in Proceedings of the IEEE, vol. 92, no. 3, 2004, pp. 401-422.

[12] R. Kan, "From Moments of Sum to Moments of Product," Journal of Multivariate Analysis, vol. 99, no. 3, pp. 542-554, March 2008. 